PROCEEDINGS OF THE

AMERICAN MATHEMATICAL SOCIETY

Volume 133, Number 6, Pages 1707-1711

S 0002-9939(04)07700-7

Article electronically published on December 6, 2004

\title{
A PROBABILISTIC PROOF OF THE FUNDAMENTAL THEOREM OF ALGEBRA
}

\author{
MIHAI N. PASCU \\ (Communicated by Richard C. Bradley) \\ I dedicate this paper to my dear friend M. K.
}

\begin{abstract}
We use Lévy's theorem on invariance of planar Brownian motion under conformal maps and the support theorem for Brownian motion to show that the range of a non-constant polynomial of a complex variable consists of the whole complex plane. In particular, we obtain a probabilistic proof of the fundamental theorem of algebra.
\end{abstract}

\section{INTRODUCTION}

The Fundamental Theorem of Algebra is a well-established result in mathematics, and there are several proofs of it in the mathematical literature. In the present paper we give a new probabilistic proof of it, as follows:

Theorem 2.4. If $f: \mathbb{C} \rightarrow \mathbb{C}, f(z)=a_{n} z^{n}+a_{n-1} z^{n-1}+\ldots+a_{1} z+a_{0}\left(a_{n} \neq 0\right.$, $\left.a_{0}, \ldots, a_{n} \in \mathbb{C}\right)$ is a polynomial of degree $n \geq 1$, then $f(\mathbb{C})=\mathbb{C}$.

In particular, the equation $f(z)=0$ has at least one root in $\mathbb{C}$.

The purpose of this paper is two-fold: on the one hand, we show how probabilistic methods can be used in order to give a simple proof of this particular result, and on the other hand, we present a method which might be suitable for deriving new properties about the range of analytic functions.

Probabilistic methods have proved to be a useful tool in Analysis. One could, for example, refer to monographs such as [2] and [3]; among others, they contain probabilistic proofs of analytic results such as: Privalov's and Plessner's Theorem (3], pp. $132-134)$, Koebe's $1 / 4$ Theorem (2], pp. 323 - 326) and Picard's Theorem (3], pp. $139-142$ or [2], pp. $320-322$ ), to mention just a few wellknown results related to the range of analytic functions (incidentally, both proofs of Picard's Theorem mentioned above have gaps; see the web site of the second author for a revised proof). For some recent activity in this area, one could see for example [4].

The present proof can also be modified to give a proof of Picard's Theorem in the particular case of a $p$-valued entire function (see the remark preceding Theorem 2.5),

Received by the editors October 10, 2003 and, in revised form, February 4, 2004.

2000 Mathematics Subject Classification. Primary 30C15; Secondary 60J65.

Key words and phrases. Brownian motion, Lévy's theorem, support theorem.

This work was supported in part by NSF grant \# 0203961 - DMS.

(C)2004 American Mathematical Society Reverts to public domain 28 years from publication 
and shows explicitly the reason for which the $p$-valuedness is a sufficient condition for an entire function to omit no values in $\mathbb{C}$.

While interesting in their own, probabilistic methods shed a new perspective on the subject and are able sometimes to provide answers to open problems in Analysis (see for example a partial resolution of the Hot Spots conjecture by probabilistic arguments in [5]).

\section{A probabilistic proof of the Fundamental Theorem of Algebra}

2.1. Preliminaries. We begin by reviewing some ingredients needed for the proof.

Recall that a complex map $f: \mathbb{C} \rightarrow \mathbb{C}$ is called entire if it is analytic in $\mathbb{C}$.

For a given integer $p \geq 1$, a complex map $f: D \rightarrow \mathbb{C}$ is said to be $p$-valued if for any $w \in \mathbb{C}$ there are at most $p$ solutions in $D$ to the equation $f(z)=w$, and there exists $w_{0} \in \mathbb{C}$ for which the equation $f(z)=w_{0}$ has exactly $p$ roots in $D$.

We recall the following result on local correspondence for analytic maps (see [1], p. 131):

Theorem 2.1. Suppose that $f(z)$ is analytic at $z_{0}, f\left(z_{0}\right)=w_{0}$ and that $f(z)-w_{0}$ has a zero of order $n$ at $z_{0}$. If $\varepsilon>0$ is sufficiently small, there exists a corresponding $\delta>0$ such that for all $w$ with $\left|w-w_{0}\right|<\delta$ the equation $f(z)=w$ has exactly $n$ roots in the disk $\left|z-z_{0}\right|<\varepsilon$.

We will need the following support theorem for Brownian motion (see [2], p. 59, for a proof), showing that with positive probability the paths of a Brownian motion follow the graph of a continuous function:

Theorem 2.2. If $\varphi:[0, t] \rightarrow \mathbb{R}^{d}$ is continuous $(d \geq 1), B_{t}$ is a d-dimensional Brownian motion starting at $B_{0}=\varphi(0)$ and $\varepsilon>0$, then there exists $c>0$ such that

$$
P^{\varphi(0)}\left(\sup _{s \leq t}\left\|B_{s}-\varphi(s)\right\|<\varepsilon\right)>c,
$$

where $c$ can be taken to depend only on $t, \varepsilon$ and the modulus of continuity of $\varphi$.

Also, we will need Lévy's theorem on conformal invariance of planar Brownian motion, showing that the image of a planar Brownian motion under an entire map is a time change of another Brownian motion (see 2, p. 310, for a proof). We have:

Theorem 2.3. Let $f: \mathbb{C} \rightarrow \mathbb{C}$ be an entire map and $B_{t}$ a 2-dimensional Brownian motion starting at $B_{0}=x$. Then $f\left(B_{\alpha_{t}}\right)$ is a 2-dimensional Brownian motion starting at $f\left(B_{0}\right)=f(x)$, where

$$
\alpha_{t}=\inf \left\{s: A_{s} \geq t\right\} \text { and } A_{t}=\int_{0}^{t}\left|f^{\prime}\left(B_{s}\right)\right|^{2} d s .
$$

Recall that given a domain $D$, a closed curve $\gamma \subset D$ is said to be homotopic to zero in $D$ if the curve $\gamma$ can be deformed continuously in $D$ to a constant curve. It is known that the homotopy is a topological property (it is preserved under continuous mappings).

We denote by $D(z, r)$ the open disk centered at $z \in \mathbb{C}$ of radius $r>0$. 
2.2. Main results. We are now ready to prove the Fundamental Theorem of Algebra, as follows:

Theorem 2.4. If $f: \mathbb{C} \rightarrow \mathbb{C}, f(z)=a_{n} z^{n}+a_{n-1} z^{n-1}+\ldots+a_{1} z+a_{0}\left(a_{n} \neq 0\right.$, $\left.a_{0}, \ldots, a_{n} \in \mathbb{C}\right)$ is a polynomial of degree $n \geq 1$, then $f(\mathbb{C})=\mathbb{C}$.

In particular, the equation $f(z)=0$ has at least one root in $\mathbb{C}$.

The idea of the proof is that if $f$ omits a certain value in $\mathbb{C}$, then by the support theorem, a planar Brownian motion will wind around this value with positive probability; if we choose this Brownian motion to be the image under $f$ of a Brownian motion in the domain of $f$, this will allow us to construct (on a set of positive probability) a closed curve $\gamma$ in the domain of $f$ that is homotopic to zero, while its image $\Gamma=f(\gamma)$ is not, thus obtaining a contradiction. Here are the details:

Proof. Assume $f$ never assumes the value $w_{0} \in \mathbb{C}$, that is, the equation $f(z)=w_{0}$ has no roots in $\mathbb{C}$.

Since $f$ is a (non-constant) polynomial of degree $n \geq 1$, there exists $p \leq n$ such that $f$ is a $p$-valent function. Therefore, for any $w \in \mathbb{C}$, the equation $f(z)=w$ has at most $p$ roots in $\mathbb{C}$, and there exists $w_{1} \in \mathbb{C}$ such that the equation $f(z)=w_{1}$ has exactly $p$ roots in $\mathbb{C}$.

By replacing $f(z)$ with

$$
\widetilde{f}(z)=\frac{f(z)-w_{0}}{w_{1}-w_{0}}
$$

if necessary, we may assume that $w_{0}=0$ and $w_{1}=1$, that is, the equation $f(z)=0$ has no roots in $\mathbb{C}$, and the equation $f(z)=1$ has exactly $p$ roots in $\mathbb{C}$.

Let $z_{1}, z_{2}, \ldots, z_{m}$ be the distinct roots of $f(z)=1$ in $\mathbb{C}$, with multiplicities $n_{1}, n_{2}, \ldots, n_{m}$ (and therefore $n_{1}+n_{2}+\ldots+n_{m}=p$ ).

By the continuity of $f$, we can choose $\varepsilon>0$ small enough so that it satisfies

$$
|f(z)-1|<\frac{1}{4} \text { for all } z \in \bigcup_{i=1}^{m} D\left(z_{i}, \varepsilon\right) .
$$

By choosing a smaller $\varepsilon>0$, if necessary, and using Theorem 2.1, it follows that there exist $\delta_{1}, \delta_{2}, \ldots, \delta_{m}>0$ such that for $|w-1|<\delta_{i}$, the equation $f(z)=w$ has exactly $n_{i}$ roots in $\left|z-z_{i}\right|<\varepsilon$, for all $i \in\{1, \ldots, n\}$. Since $n_{1}+n_{2}+\ldots+n_{m}=p$ and $f$ is $p$-valent (thus $f(z)=w$ cannot have more than $p$ roots in $\mathbb{C}$ ), it follows that these roots are all the roots of the equation $f(z)=w$ in $\mathbb{C}$. We obtained the following:

$$
|f(z)-1|<\delta \Rightarrow z \in \bigcup_{i=1}^{m} D\left(z_{i}, \varepsilon\right)
$$

where $\delta=\min \left\{\delta_{1}, \delta_{2}, \ldots, \delta_{m}\right\}$; without loss of generality, we can also assume $\delta<\frac{1}{4}$.

Consider now a Brownian motion $B_{t}$ in $\mathbb{C}$ starting at $B_{0}=z_{1}$.

Since $f$ is entire, by Theorem 2.3 it follows that $W_{t}=f\left(B_{\alpha_{t}}\right)$ is a Brownian motion starting at $f\left(B_{0}\right)=f\left(z_{1}\right)=1$, where the time change $\alpha_{t}$ is the inverse of the nondecreasing process

$$
A_{t}=\int_{0}^{t}\left|f^{\prime}\left(B_{s}\right)\right|^{2} d s .
$$


The support theorem for Brownian motion (Theorem[2.2) shows that with positive probability we have:

$$
\left|W_{t}-e^{2 \pi i t}\right|<\delta \text {, for all } t \in[0, m] .
$$

Fix now an $\omega$ in the sample space $\Omega$ for which (2.6) holds. Since $\left|f\left(B_{\alpha_{j}}(\omega)\right)-1\right|$ $=\left|W_{j}(\omega)-1\right|=\left|W_{j}(\omega)-e^{2 \pi i \cdot j}\right|<\delta$ for all $j \in\{0,1, \ldots, m\}$, and using (2.4), it follows that $B_{\alpha_{j}}(\omega) \in \bigcup_{i=1}^{m} D\left(z_{i}, \varepsilon\right)$, for all $j \in\{0,1, \ldots, m\}$.

The box principle shows that there exist $0 \leq j<k \leq m$ such that $B_{\alpha_{j}}(\omega), B_{\alpha_{k}}(\omega)$ $\in D\left(z_{l}, \varepsilon\right)$, for some $1 \leq l \leq m$.

Consider the closed curve $\gamma$ formed by concatenation of $\left[z_{l}, B_{\alpha_{j}}(\omega)\right], B_{\alpha_{t}}(j \leq$ $t \leq k)$ and $\left[B_{\alpha_{k}},(\omega), z_{l}\right]$, and let $\Gamma=f(\gamma)$ be the image of $\gamma$ under $f$ (we denoted by $[x, y]$ the straight line segment with endpoints $x$ and $y$ ).

By construction $\left[z_{l}, B_{\alpha_{j}}(\omega)\right],\left[B_{\alpha_{k}}(\omega), z_{l}\right] \subset D\left(z_{l}, \varepsilon\right) \subset \bigcup_{i=1}^{m} D\left(z_{i}, \varepsilon\right)$, and using (2.3) it follows that $f\left(\left[z_{l}, B_{\alpha_{j}}(\omega)\right]\right), f\left(\left[B_{\alpha_{k}}(\omega), z_{l}\right]\right) \subset D\left(1, \frac{1}{4}\right)$. By the choice of $\omega$ it follows that $W_{t}(\omega)=f\left(B_{\alpha_{t}}(\omega)\right), j \leq t \leq k$, lies in the $\delta$-tube about the unit circle centered at the origin, and since $\delta<\frac{1}{4}$, it follows that the index of the curve $\Gamma$, with respect to 0 is the same as the index of the curve $e^{2 \pi i t}, j \leq t \leq k$, with respect to this point:

$$
n(\Gamma, 0)=n\left(\left(e^{2 \pi i t}\right)_{j \leq t \leq k}, 0\right)=k-j \neq 0,
$$

which shows that $\Gamma$ is not homotopic to zero in $f(\mathbb{C})$ (recall that $0 \notin f(\mathbb{C})$ ).

However, this contradicts the fact that $\Gamma=f(\gamma)$ is the image under the continuous function $f$ of the curve $\gamma$, which is homotopic to zero in the domain $\mathbb{C}$ of $f$ (and should be therefore also homotopic to zero in the range of $f$ ).

The contradiction obtained shows that $f$ cannot omit the value 0 , and therefore (see the remarks at the beginning of the proof) the range of $f$ must consist of all of $\mathbb{C}$, that is, $f(\mathbb{C})=\mathbb{C}$.

A careful examination of the proof above shows that we did not explicitly use the fact that $f$ is a polynomial; the only part where we have used the fact that $f$ is a polynomial was in showing that $f$ is a $p$-valent (entire) function in $\mathbb{C}$. Therefore, the above proof also shows the following:

Theorem 2.5. If $f: \mathbb{C} \rightarrow \mathbb{C}$ is an entire p-valent function $(p \geq 1)$, then $f$ does not omit any value in $\mathbb{C}$.

Unfortunately, the class of $p$-valent entire functions coincides with the class of polynomials of degree $p$, so the above theorem is equivalent to Theorem 2.4 However, one could use the ideas in the proof above and apply them, for example, to the (larger) class of $p$-valent analytic functions in the unit disk, in order to obtain similar statements about the range of such functions.

\section{REFERENCES}

1. L. V. Ahlfors, Complex Analysis, McGraw-Hill, New York, third edition (1978). MR0510197 (80c:30001)

2. R. Bass, Probabilistic Techniques in Analysis, Springer, New York (1995). MR 1329542 (96e:60001)

3. R. Durrett, Brownian Motion and Martingales in Analysis, Wadsworth, Belmont, CA (1984). MF $0750829(87 \mathrm{a}: 60054)$ 
4. M. D. O'Neill, A geometric proof of the twist point theorem, Preprint (available at http://math.mckenna.edu/moneill).

5. M. N. Pascu, Scaling coupling of reflecting Brownian motions and the hot spots problem, Trans. Amer. Math. Soc. 354 (2002), no. 11, pp. 4681 - 4702. MR.1926894 (2003i:60141)

Department of Mathematics, Purdue University, West Lafayette, Indiana 47907-2067

E-mail address: pascu@math.purdue.edu

Current address: Faculty of Mathematics and Computer Science, "Transilvania" University of Braşov, Str. Iuliu Maniu Nr. 50, Braşov, Jud. Braşov - COD 2200, Romania

E-mail address: mihai.pascu@unitbv.ro 